\title{
Biotransformation of Monepantel in Two Species Meadow Plants (Plantago Lanceolata and Campanula Rotundifolia)
}

\author{
Lucie Raisová Stuchlíková ${ }^{1}$, Radka Podlipná $^{\mathbf{2}}$, Barbora Szotáková $^{1}$, Lenka Skálová $^{1}$ \\ ${ }^{1}$ Department of Biochemical Sciences, Faculty of Pharmacy in Hradec Králové, Charles University \\ Hradec Králové, Czech Republic \\ ${ }^{2}$ Laboratory of Plant Biotechnologies, Institute of Experimental Botany, Czech Academy of Sciences \\ Rozvojová 263, Praha 6, Czech Republic \\ lucie.raisova@faf.cuni.cz; podlipna@ueb.cas.cz
}

\section{Extended Abstract}

Monepantel (MOP) is the first molecule from the new class of aminoacetonitrile anthelmintics approved for use against nematodes in small ruminants. Contrary to other anthelmintics, MOP is also effective against nematodes strains resistant to benzimidazoles, levamisole, morantel and macrocyclic lactones. Although the use of MOP in veterinary practises has increased rapidly, at the same time they represent a risk to the environment. MOP administered to animals enter into environment primarily through its excretion in faeces or urine. Following excretion, MOP may persist in the environment and impact non-target organisms. Plants are able to uptake xenobiotics, including drugs, and detoxify them via biotransformation. However, only drug biotransformation into non-toxic and stable metabolites and their consequent accumulation in plants represent drug detoxification. For that reasons, knowledge of biotransformation pathways of drug in plants is very important.

The objectives of this study were to identify the MOP metabolites and MOP biotransformation pathways in two species meadow plants - cell suspensions (Campanula rotundifolia and Plantago lanceolata), which commonly growth on pastures. Callus culture was achieved from primary callus rising on stem segments of plant seedlings. Suspension cultures derived from this callus were grown. The suspensions were incubated with MOP $(10 \mu \mathrm{M})$ for 24 hours. Before analysis, homogenized suspensions were subjected to liquid-liquid extraction. The samples were analysed using UHPLC/MS (QqTOF) in negative-ion mode.

The results showed that enzymatic system of plantain is able to uptake, translocate and biotransform MOP into several metabolites, especially glycosides. MOP biotransformation consisted of two-step S-oxidation, hydroxylation and conjugation with glucose. Comparing both species, some differences in MOP metabolism were identified. Based on obtained results, schemes of metabolic pathway of MOP in Campanula rotundifolia and Plantaga lanceolata were proposed.

This project was supported by Czech Science Foundation, grant No. 15-05325S.

\section{Reference}

[1] H. Bártíková, L. Skálová, "Veterinary drugs in the environment and their toxicity to plants," Chemosphere, vol. 144, pp. 2290-2301, 2016. 